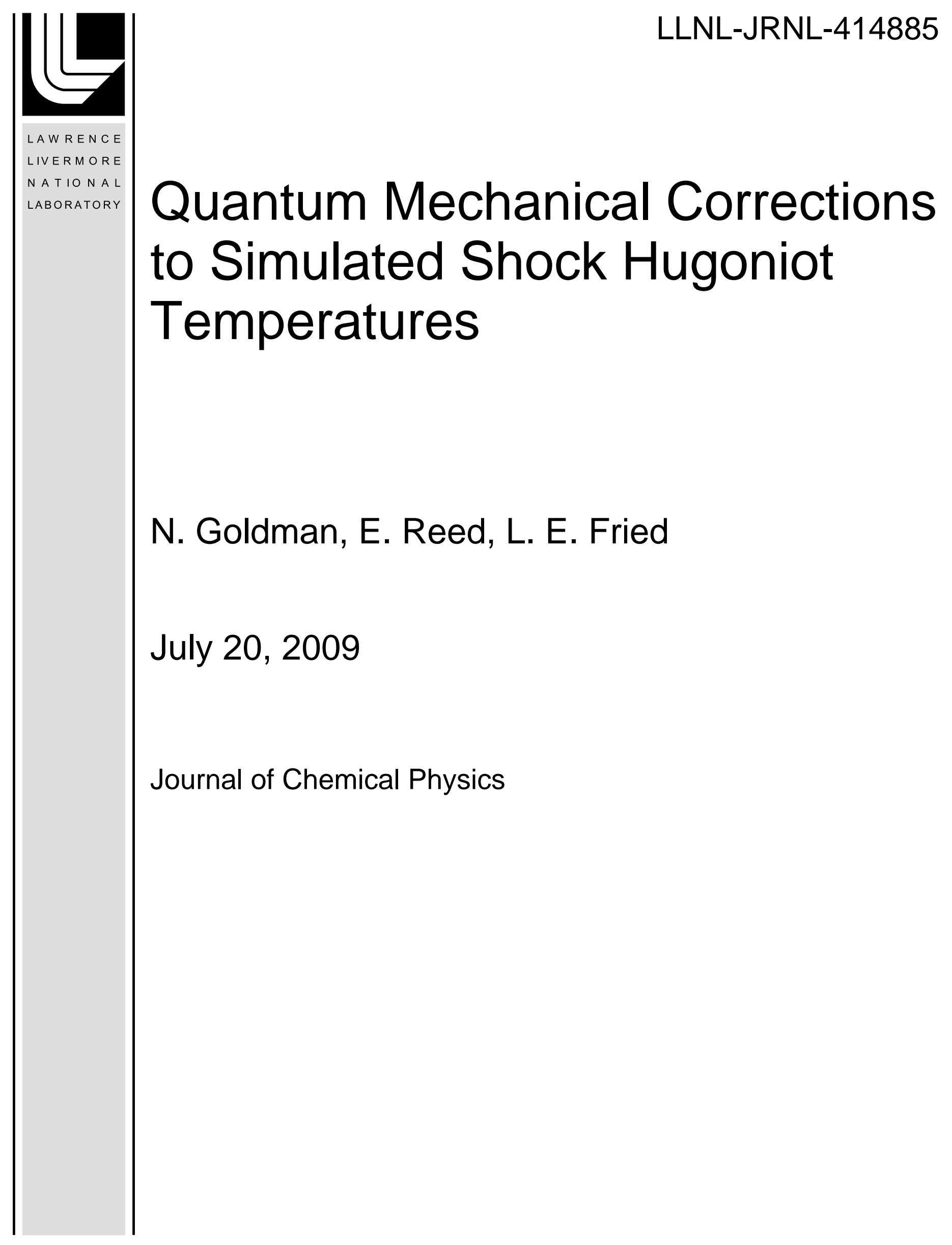


This document was prepared as an account of work sponsored by an agency of the United States government. Neither the United States government nor Lawrence Livermore National Security, LLC, nor any of their employees makes any warranty, expressed or implied, or assumes any legal liability or responsibility for the accuracy, completeness, or usefulness of any information, apparatus, product, or process disclosed, or represents that its use would not infringe privately owned rights. Reference herein to any specific commercial product, process, or service by trade name, trademark, manufacturer, or otherwise does not necessarily constitute or imply its endorsement, recommendation, or favoring by the United States government or Lawrence Livermore National Security, LLC. The views and opinions of authors expressed herein do not necessarily state or reflect those of the United States government or Lawrence Livermore National Security, LLC, and shall not be used for advertising or product endorsement purposes. 


\title{
Quantum Mechanical Corrections to Simulated Shock Hugoniot Temperatures
}

\author{
Nir Goldman, Evan J. Reed, and Laurence E. Fried \\ Chemistry, Materials, Earth and Life Sciences, \\ Lawrence Livermore National Laboratory, Livermore, California 94550
}

(Dated: July 17, 2009)

\begin{abstract}
We present a straightforward method for the inclusion of quantum nuclear vibrational effects in molecular dynamics calculations of shock Hugoniot temperatures. Using a Grüneisen equation of state and a quasi-harmonic approximation to the vibrational energies, we derive a simple, postprocessing method for calculation of the quantum corrected Hugoniot temperatures. We have used our novel technique on $a b$ initio simulations of both shock compressed water and methane. Our results indicate significantly closer agreement with all available experimental temperature data for these two systems. Our formalism and technique can be easily applied to a number of different shock compressed molecular liquids or covalent solids, and has the potential to decrease the large uncertainties inherent in many experimental Hugoniot temperature measurements of these systems.

PACS numbers:
\end{abstract}




\section{INTRODUCTION}

The equation of state of materials under extreme pressures and temperatures is of great importance for understanding planetary interiors ${ }^{1}$ as well as the chemical reactivity that occurs under strong dynamic compression ${ }^{2}$. Diamond anvil cell experiments have successfully accessed high pressure, low temperature states of matter ${ }^{3}$, as well as the lower pressure, high temperature melting line of compressed materials ${ }^{4}$. Thermodynamic states that have been inaccessible with diamond anvil cells have traditionally been achieved through shock compression. Shock compression dynamically strains the sample in one spatial dimension while simultaneously heating the sample ${ }^{5}$. However, measurement of shock Hugoniot (thermodynamic end state) temperatures of many systems remains an unresolved issue ${ }^{6}$. Although Raman scattering has been used at relatively low conditions ${ }^{7}$ (e. g., 12.1 GPa, $728 \mathrm{~K}$ ), in general pyrometric measurements are used to determine Hugoniot temperatures ${ }^{8,9}$. In this case, the measured intensity versus wavelength of radiation emitted from the shock compressed sample is fit to a greybody Planckian distribution ${ }^{9}$, with the assumption that the emissivity is independent of wavelength. Accurate temperatures remain difficult to determine due to large uncertainties in the calibration of these pyrometric measurements ${ }^{6}$. As a result, experiments tend to rely on equation of state models for temperature data, which have been shown to be inaccurate for some systems ${ }^{8}$. Molecular Dynamics (MD) simulations provide an independent route to temperature determination, where material properties such as the shock Hugoniot states are readily computed ${ }^{10-14}$.

Empirical potentials ${ }^{15,16}$ and tight-binding simulations ${ }^{10}$ have been used successfully to conduct MD simulations of the shock compression of several reactive systems. However, accurate modeling of the breaking and forming of chemical bonds usually requires the use of quantum theories such as Density Functional Theory (DFT), e. g., Ref. ${ }^{17}$. DFT has been shown to accurately reproduce the high pressure-temperature phase boundaries ${ }^{4,18}$ and shock Hugoniot properties of many materials ${ }^{12-14,19}$. However, DFT-MD simulations have been shown to under-predict experimental Hugoniot temperatures for covalently bonded materials by up to $20-30 \%{ }^{14,19}$. Molecular Dynamics simulations in general propagate classical nuclear equations of motion, neglecting quantum zero-point and vibrational energy effects. In particular, many covalently bonded systems have bond vibrational frequencies that are only excited out of their quantum ground state at high ionic temperatures, such as 
in liquid water, where the Debeye temperature is approximately 5000 K. Quantum effects at ionic temperatures close to this value yield a true heat capacity of the system that is likely significantly lower than the classical limit. As a result, the erroneous classical heat capacities inherent in MD simulations of these materials could produce Hugoniot temperatures that are too low. Determination of material equations of state could be greatly facilitated by a more accurate MD temperature calculation methodology that includes these quantum effects.

In this work, we report a novel theoretical post-processing methodology for the inclusion of quantum nuclear vibrational effects in the equation of state from MD simulations of shock compression. Our technique is based upon computation of the velocity autocorrelation function and is independent of the force-field used in the simulation. We test our theory by computing quantum corrected Hugoniot temperatures from DFT-MD simulations of both shock compressed water and methane. Our results show that our method yields improved agreement with all available experimental data. We then analyze the power spectra from both systems in order to create a simple physical picture for the magnitude of the quantum correction at higher temperatures and pressures. Our calculations represent the first time these quantum effects have been included in MD Hugoniot temperatures and have been quantified, to date.

\section{METHODS}

In order to derive a method for calculation of the quantum nuclear vibration effects on the Hugoniot temperature, we derive expressions relating the thermal energies for the classical and quantum systems. We first approximate the equation of state of the true (quantum) system by using a Grüneisen equation of $\operatorname{state}^{5}$ where the pressure and internal energy are divided into "cold" and "thermal" parts, dependent on the system volume $V$ and temperature $T:$

$$
\begin{gathered}
E(V, T)=E_{c}(V)+E_{T}(V, T) \\
P(V, T)=P_{c}(V)+P_{T}(V, T)
\end{gathered}
$$

Here, the quantities $E_{c}$ and $P_{c}$ correspond to the cold components of the internal energy and pressure, respectively, which are independent of temperature. $E_{T}(V, T)$ corresponds to 
the thermal component of the internal energy. The thermal component of the pressure is defined as $P_{T} \equiv \Gamma(V) E_{T}(V, T) / V$, where $\Gamma(V)$ is the dimensionless Grüneisen coefficient, defined as $\Gamma(V) \equiv-\partial \ln \bar{\nu} / \partial \ln V$. Here, $\bar{\nu}$ is the average vibron frequency. The cold and thermal portions of the internal energy can be written in terms of their electronic $(e l)$ and ionic $(i)$ components:

$$
\begin{gathered}
E_{c}(V)=E_{c}^{e l}(V)+E_{c}^{i}(V) \\
E_{T}(V)=E_{T}^{i}(V, T)+E_{T}^{e l}(V, T) .
\end{gathered}
$$

For this work, our MD simulations are all in the electronic ground-state, i. e., $E_{c}^{e l}(V)$, which allows us to neglect electronic contributions to the thermal energy $\left(E_{T}^{e l}(V, T)=0\right)$. Regardless, we include $E_{T}^{e l}(V, T)$ in our formalism, although our final expression for the difference between the quantum and classical thermal energy will depend exclusively on the ionic component.

In first principles Molecular Dynamics, the ions are treated classically, and we denote the equation of state for the classical system with lower case letters:

$$
\begin{gathered}
e(V, t)=e_{c}(V)+e_{T}(V, t) \\
p(V, t)=p_{c}(V)+\Gamma(V) e_{T}(V, t) / V,
\end{gathered}
$$

where $e, p$, and $t$ represent the classical internal energy, pressure and temperature, respectively, we have substituted in the definition of the thermal component of the pressure in Eqn. 6, and $\Gamma(V)$ is the same Grüneisen coefficient, mentioned above. Here, the volumes of the quantum and classical systems are constrained to be the same. For the classical system the cold energy is entirely electronic, viz.,

$$
e_{c}(V)=E_{c}^{e l}(V)
$$

Inclusion of electronic thermal effects yields the following for the classical thermal energy:

$$
e_{T}(V, t)=e_{T}^{i}(V, t)+E_{T}^{e l}(V, T)
$$

We now assume that the vibrational density of states and electronic energies $\left(E_{c}^{e l}\right.$ and $\left.E_{T}^{e l}\right)$ are the same in both systems. Consequently, the Grüneisen coefficients are also the 
same for both classical and quantum systems. We constrain both systems to have the same initial pressure $P_{0}$ and temperature $T_{0}$. We ignore the contribution of zero-point vibrations to the cold component of the pressure $P_{c}^{i}(V)$, which we estimate to be smaller than the error bars in our computed stress tensors (see below). As a result, we have $p_{c}(V)=P_{c}(V)$.

For a shock compressed process, both quantum and classical systems will satisfy the Hugoniot relation:

$$
\begin{gathered}
E(V, T)-E\left(V_{0}, T_{0}\right)=\frac{1}{2}\left(V_{0}-V\right)\left(P+P_{0}\right) \\
e(V, t)-e\left(V_{0}, T_{0}\right)=\frac{1}{2}\left(V_{0}-V\right)\left(p+P_{0}\right) .
\end{gathered}
$$

Using equations 1 and 2, we can then expand equation 9 as the following:

$$
E_{c}(V)-E_{c}\left(V_{0}\right)+E_{T}(V, T)-E_{T}\left(V_{0}, T_{0}\right)=\frac{1}{2}\left(V_{0}-V\right)\left[P_{c}(V)+\frac{\Gamma(V) E_{T}(V, T)}{V}+P_{0}\right] .
$$

Expanding the cold energy $E_{c}(V)$ and the thermal energy $E_{T}(V, T)$ into their electronic and ionic parts, and solving for $E_{T}^{i}(V, T)$, we obtain:

$$
E_{T}^{i}(V, T)=\frac{-E_{c}^{e l}(V)+E_{c}^{e l}\left(V_{0}\right)-E_{c}^{i}(V)+E_{c}^{i}\left(V_{0}\right)+\frac{1}{2}\left(P_{c}(V)+P_{0}\right)\left(V_{0}-V\right)-E_{T}^{e l}(V, T)+E_{T}\left(V_{0}, T_{0}\right)}{\left[1-\frac{\Gamma(V)\left(V_{0}-V\right)}{2 V}\right]} .
$$

Similarly, for the classical system, we obtain:

$$
e_{T}^{i}(V, t)=\frac{-E_{c}^{e l}(V)+E_{c}^{e l}\left(V_{0}\right)+\frac{1}{2}\left(P_{c}(V)+P_{0}\right)\left(V_{0}-V\right)-E_{T}^{e l}(V, T)+e_{T}\left(V_{0}, T_{0}\right)}{\left[1-\frac{\Gamma(V)\left(V_{0}-V\right)}{2 V}\right]} .
$$

We then subtract Eqns. 12 and 13 to obtain the following relation for $E_{T}^{i}(V, T)$ :

$$
E_{T}^{i}(V, T)=e_{T}^{i}(V, t)+\frac{\left[E_{T}^{i}\left(V_{0}, T_{0}\right)-e_{T}^{i}\left(V_{0}, T_{0}\right)\right]-\left[E_{c}^{i}(V)-E_{c}^{i}\left(V_{0}\right)\right]}{\left(1-\frac{\Gamma(V)\left(V_{0}-V\right)}{2 V}\right)}
$$

which we simplify to

$$
E_{T}^{i}(V, T)=e_{T}^{i}(V, t)+\Delta E\left(T_{0}, V_{0} ; V\right)
$$

where $\Delta E\left(T_{0}, V_{0} ; V\right)$ equals the second term on the right hand side of Eqn. 14.

In order to compute the values of the cold and thermal quantum ionic energies, $E_{c}^{i}(V)$ and $E_{T}^{i}(V, T)$, we apply the quasi-harmonic approximation to the vibrational states of the system. As a result, we write the cold ionic energies as: 


$$
E_{c}^{i}(V)=\frac{1}{2} \int_{0}^{\infty} \rho(\omega, V) \hbar \omega d \omega
$$

where $\rho(\omega, V)$ is the vibrational density of states of the system, which we take to be the power spectrum of the time dependent velocity autocorrelation function, viz., $\rho(\omega, V) \propto$ $\int_{0}^{\infty} d \tau \cos (\omega, \tau)\langle\mathbf{v}(\tau) \mathbf{v}(0)\rangle /\left\langle\mathbf{v}(0)^{2}\right\rangle$. We normalize $\rho(\omega, V)$ to $\int_{0}^{\infty} d \omega \rho(\omega, V)=3 N_{A}$, where $N_{A}$ equals Avogadro's Number.

Similarly, we write for $E_{T}^{i}(V, T)$ :

$$
E_{T}^{i}(V, T)=\int_{0}^{\infty} d \omega E_{Q_{H O}}^{T}(\omega, T), \rho(\omega, V)
$$

where $E_{Q_{H O}}^{T}(\omega, T)$ is the average thermal energy of the quantum harmonic oscillator:

$$
E_{Q_{H O}}(\omega, T)=\frac{\hbar \omega}{e^{\hbar \omega / k_{B} T}-1} .
$$

For a classical quasi-harmonic material, we have the standard expression for the thermal ionic energy, $e_{T}^{i}(V, t)=3 N k_{B} t$. The low frequency part of the vibrational spectrum $(\nu<$ $T$ ) for a molecular liquid exhibits nearly classical behavior. Hence, our quantum energy correction will be significant only for the higher frequency, solid-like modes of the system, consistent with the quasi-harmonic approximation.

We now relate $E_{T}^{i}(V, T)$ and $e_{T}^{i}(V, t)$ by taking Eqns. 17 and 15 to form:

$$
E_{T}^{i}(V, T)=\int_{0}^{\infty} d \omega E_{Q_{H O}}(\omega, T) \rho(\omega, V)=3 N k_{B} t+\Delta E\left(T_{0}, V_{0} ; V\right) .
$$

We can form an expression for the quantum correction to the thermal energy as a function of frequency by solving for $\Delta E\left(T_{0}, V_{0} ; V\right)$ and inserting classical energy into the integrand:

$$
\Delta E\left(T_{0}, V_{0} ; V\right)=\int_{0}^{\infty} d \omega \rho(\omega, V)\left(\frac{\hbar \omega}{e^{\hbar \omega / k_{B} T}-1}-k_{B} t\right)
$$

Consequently, given the temperature of the quantum $(T)$ and classical systems $(t)$, we can determine the contribution to the correction from specific vibrational modes for a given shock compressed system.

Thus, determination of the Hugoniot quantum temperature proceeded as follows. After calculation of the power spectrum, we use Eqns. 16 and 17 to determine the value of $\Delta E\left(T_{0}, V_{0} ; V\right)$. Values of $\Gamma(V)$ were computed by first performing two constant volumetemperature (NVT) simulations at the Hugoniot temperature and at $200 \mathrm{~K}$ above for both 
the lowest and highest simulation densities for each system. $\Gamma(V)$ was then determined by using the relation $\Gamma(V)=V \partial P / \partial E \approx V \Delta P / \Delta E$. Values for water of $\Gamma(V)$ at densities between the two extrema were computed via linear regression. Our approximation for $\Gamma(V)$ is discussed further in the Results section, below. We then use Eqn. 19 to solve numerically for the quantum Hugoniot temperature, which we label $T_{Q M}$. Since the power spectrum can be calculated for any saved simulation trajectory, our technique can easily be applied to any previously computed shock compression MD simulation.

It is possible to compute a quantum Hugoniot pressure correction from the definition of the thermal pressure $P_{T}$, i. e., $\Delta P=\Gamma(V) \Delta E\left(V_{0}, T_{0} ; V\right) / V$. However, for all of our simulations the values of $\Delta P$ were less than $1 \mathrm{MPa}$, i. e., three orders of magnitude smaller than the error in the classically computed pressure. We have also estimated the contribution of zero-point vibrations to the cold component of the pressure, $P_{c}^{i}(V)=-d E_{c}^{i}(V) / d V$, by fitting our results for $E_{c}^{i}(V)$ to a simple functional form. Similarly, we find this quantum pressure correction to be less than $1 \mathrm{MPa}$.

\section{COMPUTATIONAL DETAILS}

All shock compression simulations were conducted with the Multi-Scale Shock Technique $(\mathrm{MSST})^{12,13,20-22}$. MSST is a simulation methodology based on the Navier-Stokes equations for compressible flow. Instead of simulating a shock wave within a large computational cell with many atoms, the MSST computational cell follows a Lagrangian point through the shock wave. This is accomplished by time-evolving equations of motion for the atoms and volume of the computational of cell to constrain the stress in the propagation direction to the Rayleigh line and the energy of the system to the Hugoniot energy condition ${ }^{20,22}$. For a given shock speed, these two relations describe a steady planar shock wave within continuum theory. The MSST technique thus enables simulation of the shock wave with small system sizes $^{12}$, making it possible to simulate with DFT or other computationally intensive force fields. MSST has been used in conjunction with DFT-MD to accurately reproduce the shock Hugoniot of a number of systems ${ }^{13,14}$. MSST has also been shown to accurately reproduce the sequence of thermodynamic states throughout the reaction zone of shock compressed explosives with analytical equations of $\operatorname{state}^{22}$.

All of our DFT-MD simulations for water and methane were between $5-11$ ps in length, 
with an average drift from the Hugoniot energy condition of $0.5 \%$ or less. A fictitious box mass of $3.5 \times 10^{6}$ a. u. and a wavefunction convergence criteria of $10^{-6}$ a. u. were used for all simulations unless otherwise noted. Our simulations of shock compressed water have been discussed in a previous publication ${ }^{14}$. For this system we performed simulations of the following shock velocities $(\mathrm{km} / \mathrm{s}): 5,6.5,7.5,9,10,11$ using the CPMD simulation software package ${ }^{23}$. Stronger shock velocities resulted in electronic excitation beyond the Born-Oppenheimer state, which requires the inclusion of electron thermal excitations ${ }^{24,25}$. In these simulations we used a planewave cutoff of 120 Rydberg was used with the Becke-LeeYang-Parr exchange-correlation functional ${ }^{26,27}$. Tests with the PBE exchange-correlation functional ${ }^{28}$ yielded consistent results over the pressure-temperature range of our calculations. An initial configuration of $64 \mathrm{H}_{2} \mathrm{O}$ molecules was generated from an equilibrated CPMD simulation conducted at $300 \mathrm{~K}$ with computational-cell lattice vectors of $a=19.72$ $\AA, b=9.86 \AA$, and $c=9.86 \AA$. This corresponds to the ambient density of $1 \mathrm{~g} / \mathrm{cc}$, similar to initial conditions of experiments ${ }^{29}$. Uniaxial compression of the shock wave occurred along the $a$ lattice vector. Convergence tests with up to 128 molecules showed that a system size of 32 molecules provided sufficient convergence of the stress tensor for the shock compressed configurations. Simulations at 10 and $11 \mathrm{~km} / \mathrm{s}$ had an additional force convergence criteria of $10^{-7}$ a. u.

Our simulations of shock compressed methane were conducted with the CP2K mixed basis set DFT simulation software suite ${ }^{30,31}$. We simulated shock velocities of 11 and 12.2 $\mathrm{km} / \mathrm{s}$ in order to match the small experimental data set for the methane shock temperatures as closely as possible. For these simulations we used a planewave cutoff of 280 Ry and an optimized TZVP basis set for both carbon and hydrogen, although we observed the system energy and stress tensor to be converged with the smaller DZVP basis set. We employed Goedecker-Teter-Hutter pseudopotentials ${ }^{32}$ with the PBE exchange correlation functional ${ }^{28}$. An initial configuration of $\mathrm{CH}_{4}$ molecules was generated from an equilibrated CP2K simulation conducted at $111 \mathrm{~K}$ with computational-cell lattice vectors of $a=25.06$ $\AA, b=12.53 \AA$, and $c=12.53 \AA$. Uniaxial compression of the shock wave occurred along the $a$ lattice vector. These cell dimensions yield a density of $0.432 \mathrm{~g} / \mathrm{cc}$, similar to initial conditions of experiments ${ }^{33,34}$. 


\section{RESULTS}

\section{A. Water}

Our results for the Hugoniot temperature values for water are shown in Table I and Fig. 1. We have shown excellent agreement of our simulations with experimental results for the pressure vs. density equation of state of water ${ }^{14}$. We find that our quantum mechanical corrections bring our calculated temperatures in excellent agreement with experiment. Our quantum Hugoniot temperatures provide excellent validation of DFT over a wide range of thermodynamic conditions. Our results show that quantum nuclear vibrational effects play a significant role in water even at elevated temperatures (> $4000 \mathrm{~K})$.

Using the method for approximation for $\Gamma(V)$ mentioned above, we computed a values of $\Gamma=0.681$ at $8.3 \mathrm{GPa}(5 \mathrm{~km} / \mathrm{s})$ and $\Gamma=0.355$ at $67.8 \mathrm{GPa}(11 / \mathrm{kms})$. For comparison, we compute values of $\Gamma$ from a recent equation of state for water ${ }^{35}$ of 0.990 and 0.400 , respectively. In order to examine the dependence of $E_{T}(V, T)$ on $\Gamma$, we can expand Eqn. 14 about $\Gamma_{0}$ to first order to obtain the change in $E_{T}$ as a function of $\Gamma$ :

$$
\Delta E_{T}\left(\Gamma-\Gamma_{0}\right)=E_{T}(\Gamma)-E_{T}\left(\Gamma_{0}\right)=\frac{\Delta E\left(V_{0}, T_{0} ; V\right)\left(V_{0}-V\right)\left(\Gamma-\Gamma_{0}\right)}{(2 V)\left[1-\frac{\Gamma_{0}\left(V_{0}-V\right)}{2 V}\right]^{2}}+\mathcal{O}^{2}(\Gamma)
$$

We then use our equation of state results for $\Gamma$ as an upper limit to obtain a $7.3 \%$ difference in $E_{T}$ at $8.3 \mathrm{GPa}$ and a $0.1 \%$ difference at $67.8 \mathrm{GPa}$. This results in a $5.4 \%$ and $0.1 \%$ change in the value of $T_{Q M}$, respectively. Thus, our results are relatively insensitive to the chosen value of $\Gamma$, and our method for determining its value is valid for our simulations.

In order to quantify the quantum temperature correction from specific vibrational modes, we have plot the results for $\Delta E\left(V_{0}, T_{0} ; V\right)$ from Eqn. 20 for our simulation at $T_{Q M}=584$ K (Fig. 2). At frequencies below $T_{Q M}$, we observe that the difference between the classical and quantum thermal energies is slightly positive. This is due to the fact that $T_{Q M}$ is higher in value than the classical temperature, and the vibrational modes at these low frequencies are mainly classical in nature, including the zero-frequency part of the power spectrum due to self-diffusion. However, as the frequency increases above $T_{Q M}$, we observe a significant correction due to the quantization of the vibrational energies. This confirms the validity of our treatment of the liquid molecular vibrations with a quantum quasi-harmonic 
approximation. Only the higher frequency modes above the system temperature experience significant quantum effects.

The significant quantum Hugoniot temperature correction at high shock velocity (e. g., $11 \mathrm{~km} / \mathrm{s}$ ) can be explained through examination of the computed power spectra (Fig. 3). We observe that the vibron (O-H stretch mode) broadens significantly and red-shifts as a function of increasing temperature and pressure due to a weakening of the $\mathrm{O}-\mathrm{H}$ bonds. In addition, the integrated intensity of the vibron peak at $4244 \mathrm{~K}(11 \mathrm{~km} / \mathrm{s})$ is approximately $70 \%$ of the value at ambient conditions, despite the high degree of dissociation of water under these conditions ${ }^{14}$. This allows for an increased number of vibrational states to be accessed by the system at higher pressure and temperature. The $T_{Q M}$ value of water at $4244 \mathrm{~K}$ is roughly equal to its Debeye temperature. As a result, low lying excited vibrational states will have significant contributions to the heat capacity of the system at these conditions. We calculate a quantum correction of $34.6 \%$ at a $T_{Q M}$ value of $584 \mathrm{~K}$, which decreases to a value of $16.1 \%$ at $4244 \mathrm{~K}$. This is consistent with the requirement that the quantum heat capacity must converge to the classical limit with increasing temperature.

\section{B. Methane}

We present the simulated Hugoniot values for methane in Table II and Figs. 4 and 5. We use values of $\Gamma$ of 0.438 at $32.4 \mathrm{GPa}(1 \mathrm{~km} / \mathrm{s})$ and 0.300 at $42.2 \mathrm{GPa}(12.2 \mathrm{~km} / \mathrm{s})$. Our simulations provide quantitative validation of the DFT-Generalized Gradient Approximation equation of state for methane up to pressures up to ca. $42 \mathrm{GPa}$ (Fig 4). The error in the density is most likely much lower than that at ambient conditions ${ }^{36}$ due to the decreased importance of van der Waals interactions at extreme conditions. Similar to water, we find that inclusion of quantum nuclear vibrational effects results in notable improvement between simulation and experimental results for the Hugoniot temperature (Fig. 5). Comparison of power spectra at $T_{Q M}$ values of $3244 \mathrm{~K}(32.4 \mathrm{GPa}, 11 \mathrm{~km} / \mathrm{s})$ and $4059 \mathrm{~K}(42.2 \mathrm{GPa}, 12.2$ $\mathrm{km} / \mathrm{s}$ ) to that from the initial conditions (Fig 6) shows that the methane vibron $(\mathrm{C}-\mathrm{H}$ stretch mode) broaden but red-shifts only slightly. This indicates that the $\mathrm{C}-\mathrm{H}$ bond is not significantly weakened under the conditions of our simulations. The integrated intensity of the vibron peak at both $T_{Q M}$ values of $3244 \mathrm{~K}$ and $4059 \mathrm{~K}$ is approximately equal to the value at the initial conditions, consistent with minimal methane dissociation, discussed 
below. We observe that the $T_{Q M}$ values are approximately equal to the Debeye temperature, in accordance with the significant computed quantum corrections to the temperature.

We have analyzed the chemical species present in our simulations using previously established criteria for high pressure-temperature systems ${ }^{1,14,37,38}$. Bond cutoff values $r_{c}$ were chosen based on the maximum of the potential of mean force, e. g., $W\left(R_{C H}\right)=$ $-k_{B} T \ln \left[g\left(R_{C H}\right)\right]$. We have chosen a value of $r_{c}=1.25 \AA$ for $\mathrm{C}-\mathrm{H}$ bonds in all of our simulations. In addition, in order to avoid counting species that were entirely transient and not chemically bonded ${ }^{37}$, we also chose a lifetime cutoff of 25 fs (e. g., two oscillations of a $\mathrm{C}-\mathrm{H}$ bond vibration). This criteria is intuitive since $\mathrm{C}-\mathrm{H}$ bonds with this lifetime could conceivably be detected spectroscopically.

We do not observe any $\mathrm{C}-\mathrm{C}$ or $\mathrm{H}-\mathrm{H}$ bonding in our simulations, similar to tight-binding studies at similar conditions ${ }^{10}$. Previous DFT-MD simulations observed methane decomposition at significantly higher pressures and temperatures ${ }^{39}$. At a $T_{Q M}$ of $3244 \mathrm{~K}$, we observed approximately $1 \%$ of the methane dissociated via a unimolecular mechanism, viz., $\mathrm{CH}_{4} \leftrightarrow$ $\mathrm{H}^{+}+\mathrm{CH}_{3}^{-}$. The dissociation remains unimolecular and increases to $10 \%$ when the simulation is shock compressed to $4059 \mathrm{~K}$. This small degree of dissociation is consistent with short-time scale shock compression experiment measurements of low electrical conductivity of methane under these conditions ${ }^{34}$. Diamond Anvil Cell experiments at orders of magnitude longer time scales observe diamond formation at similar conditions ${ }^{40}$. The percent change in our quantum corrected Hugoniot computed temperatures decreases from $32.5 \%$ at $11 \mathrm{~km} / \mathrm{s}$ to $25 \%$ at $12.2 \mathrm{~km} / \mathrm{s}$. Thus we observe that methane exhibits quantum corrections to the Hugoniot temperature that are equivalent to differences between simulation and experiment for water and other systems ${ }^{19}$.

\section{CONCLUSIONS}

Our quantum Hugoniot temperature calculation method yields an improved agreement between DFT-MD simulations and pyrometry experiments. Our results provide validation of DFT with the Generalized Gradient Approximation for the high pressure-temperature equation of state of both water and methane. We predict significant quantum corrections to the Hugoniot temperatures of our simulations, due to quantization of the high frequency vibron of covalently bonded systems such as water and methane. Our technique can be easily 
applied to any number of systems and can be used to decrease experimental uncertainties in shock Hugoniot temperature measurements. Our quantum Hugoniot temperature method can also be used in conjunction with MD simulations to compute experimental Hugoniot temperatures where equation of state modeling is known to be inaccurate.

Prior to our submission, we were made aware of recent results from a somewhat similar technique used to calculate the influence of quantum nuclear vibrational effects on the equation iof state of water ${ }^{41}$. This technique differs from ours in that it requires calculation of the mole fraction of non-dissociated water molecules and does not require explicit computation of the vibrational density of states. In particular, our method does not require explicit determination of the species present in the simulation. However, their results show similarly improved agreement to experiment for the Hugoniot tmeperatures.

\section{ACKNOWLEDGEMENTS}

This work was performed under the auspices of the U.S. Department of Energy by Lawrence Livermore National Laboratory under Contract DE-AC52-07NA27344. The project 06-ERD-037 was funded by the Laboratory Directed Research and Development Program at LLNL. Computations were performed at LLNL using the following massively parallel computers: prism, MCR, Thunder, uP, and Blue Gene L (BG/L). 
1 N. Goldman, L. E. Fried, I.-F. W. Kuo, and C. J. Mundy, Phys. Rev. Lett. 94, 217801 (2005).

2 R. Jeanloz, P. M. Celliers, G. W. Collins, J. H. Eggert, K. K. M. Lee, R. S. McWilliams, S. Brygoo, and P. Loubeyre, Proc. Natl. Acad. Sci. USA 104, 9172 (2007).

3 A. F. Goncharov, V. V. Struzkhin, M. S. Somayazulu, R. J. Hemley, and H. K. Mao, Science 273, 218 (1996).

4 A. F. Goncharov, N. Goldman, L. E. Fried, J. C. Crowhurst, I.-F. W. Kuo, C. J. Mundy, and J. M. Zaug, Phys. Rev. Lett. 94, 125508 (2005).

5 Y. B. Zel'dovitch and Y. P. Raizer, Physics of Shock Waves and High-Temperature Hydrodynamic Phenomena (Dover Publications, Mineola, New York, 2002).

${ }^{6}$ K. K. M. Lee, L. R. Benedetti, R. Jeanloz, P. M. Celliers, J. H. Eggert, D. G. Hicks, S. J. Moon, A. Mackinnon, L. B. D. Silva, D. K. Bradley, W. Unites, and G. W. Collins, J. Chem. Phys. 125, 014701 (2006).

7 G. I. Pangilinan and Y. M. Gupta, J. Appl. Phys. 70, 967 (1997).

8 G. A. Lyzenga, T. J. Ahrens, W. J. Nellis, and A. C. Mitchell, J. Chem. Phys. 76, 6282 (1982).

9 H. B. Radousky and A. C. Mitchell, Rev. Sci. Instrum. 60, 3707 (1989).

10 J. D. Kress, S. R. Bickham, L. A. Collins, B. L. Holian, and S. Goedecker, Phys. Rev. Lett. 83, $3896(1999)$.

11 K. Kadau, T. C. Germann, P. S. Lomdhal, and B. L. Holian, Science 296, 1681 (2002).

12 E. J. Reed, M. R. Manaa, L. E. Fried, K. R. Glaesemann, and J. D. Joannopoulos, Nature Physics 4, 72 (2008).

13 C. J. Mundy, A. Curioni, N. Goldman, I.-F. Kuo, E. Reed, L. E. Fried, and M. Ianuzzi, J. Chem. Phys. 128, 184701 (2008).

14 N. Goldman, E. J. Reed, I.-F. W. Kuo, L. E. Fried, C. J. Mundy, and A. Curioni, J. Chem. Phys. 130, 124517 (2009).

15 A. Strachan, A. C. T. van Duin, D. Chakraborty, S. Dasgupta, and W. A. Goddard, Phys. Rev. Lett. 91, 098301 (2003).

16 A. V. Bolesta, L. Zheng, D. L. Thompson, and T. D. Sewell, Phys. Rev. B 76, 224108 (2007).

17 F. Gygi and G. Galli, Phys. Rev. B 65, 220102 (2002).

18 E. Schwegler, M. Sharma, F. Gygi, and G. Galli, Proc. Nat. Acad. Sci.(USA) 105, 14779 (2008). 
19 J. D. Kress, S. Mazevet, L. A. Collins, and W. W. Wood, Phys. Rev. B 63, 024203 (2000).

20 E. J. Reed, L. E. Fried, and J. D. Joannopoulos, Phys. Rev. Lett. 90, 235503 (2003).

21 E. Reed, L. E. Fried, M. R. Manaa, and J. D. Joannopoulos, in Chemistry at Exteme Conditions, edited by M. Manaa (Elsevier, ADDRESS, 2005).

22 E. J. Reed, L. E. Fried, W. D. Henshaw, and C. M. Tarver, Phys. Rev. E 74, 056706 (2006).

23 R. Car and M. Parrinello, Phys. Rev. Lett. 55, 2471 (1985); CPMD code, version 3.11.1, Copyright IBM Corp 1990-2006, Copyright MPI für Festkörperforschung Stuttgart 1997-2001.

24 T. R. Mattsson and M. P. Desjarlais, Phys. Rev. Lett. 97, 017801 (2006).

25 M. French, T. R. Mattsson, N. Nettelman, and R. Redmer, Phys. Rev. B 97, 054107 (2009).

26 A. D. Becke, Phys. Rev. A 38, 3098 (1988).

27 C. Lee, W. Yang, and R. G. Parr, Phys. Rev. B 37, 785 (1988).

28 J. P. Perdew, K. Burke, and M. Ernzerhof, Phys. Rev. Lett. 78, 1396 (1997).

29 R. Chau, A. C. Mitchell, R. W. Minich, and W. J. Nellis, J. Chem. Phys 114, 1361 (2001).

30 J. VandeVondele, M. Krack, F. Mohamed, M. Parrinello, T. Chassaing, and J. Hutter, Comp. Phys. Comm. 167, 103 (2005).

31 CP2K code, http://cpk2.berlios.de (Copyright CP2K developers group 2000-2009).

32 S. Goedecker, M. Teter, and J. Hutter, Phys. Rev. B 54, 1703 (1996).

33 W. J. Nellis, F. H. Rhee, M. van Thiel, and A. C. Mitchell, J. Chem. Phys. 75, 3055 (1981).

34 H. B. Radousky, A. C. Mitchell, and W. J. Nellis, J. Chem. Phys. 93, 8235 (1990).

35 S. Bastea and L. E. Fried, J. Chem. Phys. 128, 174502 (2008).

36 M. J. McGrath, J. I. Siepmann, I.-F. W. Kuo, C. J. Mundy, J. VandeVondele, J. Hutter, F. Mohamed, and M. Krack, ChemPhysChem 6, 1894 (2005).

37 N. Goldman and L. E. Fried, J. Chem. Phys. 125, 044501 (2006).

38 N. Goldman and L. E. Fried, J. Chem. Phys. 126, 134505 (2007).

39 F. Ancilotto, G. L. Chiarotti, S. Scandolo, and E. Tosatti, Science 275, 1288 (1997).

40 L. R. Benedetti, J. H. Nguyen, W. A. Caldwell, H. Liu, M. Kruger, and R. Jeanloz, Science 286, 100 (1999).

41 M. French and R. Redmer, J. Phys.: Condens. Matter, submitted. 
Table I: Table of final thermodynamic for our water shock compression simulations. Error bars were determined by calculating the standard deviation over four time blocks.

\begin{tabular}{cccc}
\hline \hline Shock velocity $(\mathrm{km} / \mathrm{s})$ & Pressure $(\mathrm{GPa})$ & $\mathrm{T}_{C l}(\mathrm{~K})$ & $\mathrm{T}_{Q M}(\mathrm{~K})$ \\
\hline 5 & $8.3 \pm 0.1$ & $434 \pm 14$ & 584 \\
6.5 & $18.2 \pm 0.2$ & $791 \pm 7$ & 1047 \\
7.5 & $26.5 \pm 0.4$ & $1167 \pm 4$ & 1517 \\
9 & $42.0 \pm 0.3$ & $1995 \pm 8$ & 2480 \\
10 & $53.8 \pm 0.3$ & $2744 \pm 10$ & 3292 \\
11 & $67.8 \pm 0.2$ & $3654 \pm 6$ & 4244 \\
\hline \hline
\end{tabular}

Table II: Table of final thermodynamic states for methane shock compression simulations. Error bars were determined by calculating the standard deviation over four time blocks.

\begin{tabular}{ccccc}
\hline \hline Shock velocity $(\mathrm{km} / \mathrm{s})$ & Pressure $(\mathrm{GPa})$ & Density $\left(\mathrm{g} / \mathrm{cm}^{3}\right)$ & $\mathrm{T}_{C l}(\mathrm{~K})$ & $\mathrm{T}_{Q M}(\mathrm{~K})$ \\
\hline 11 & $32.4 \pm 0.4$ & $1.12 \pm 0.01$ & $2448 \pm 10$ & 3244 \\
12.2 & $42.2 \pm 0.3$ & $1.2 \pm 0.01$ & $3229 \pm 8$ & 4059 \\
\hline \hline
\end{tabular}




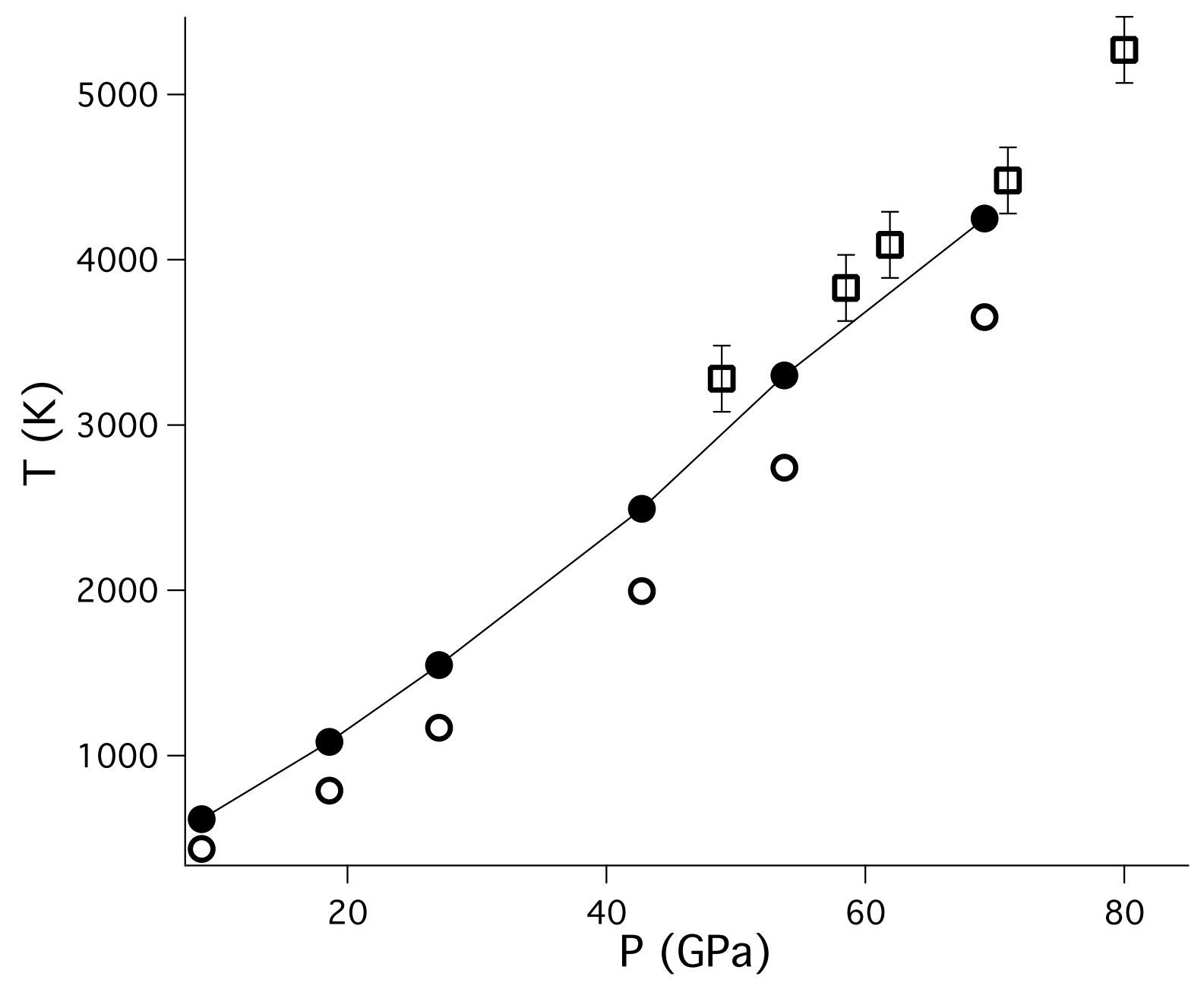

Figure 1: Plot of classical and quantum Hugoniot temperatures for water. Open circles correspond to classical ionic temperatures, solid circles to quantum corrected temperatures, and open squares to experimental results ${ }^{8}$. 


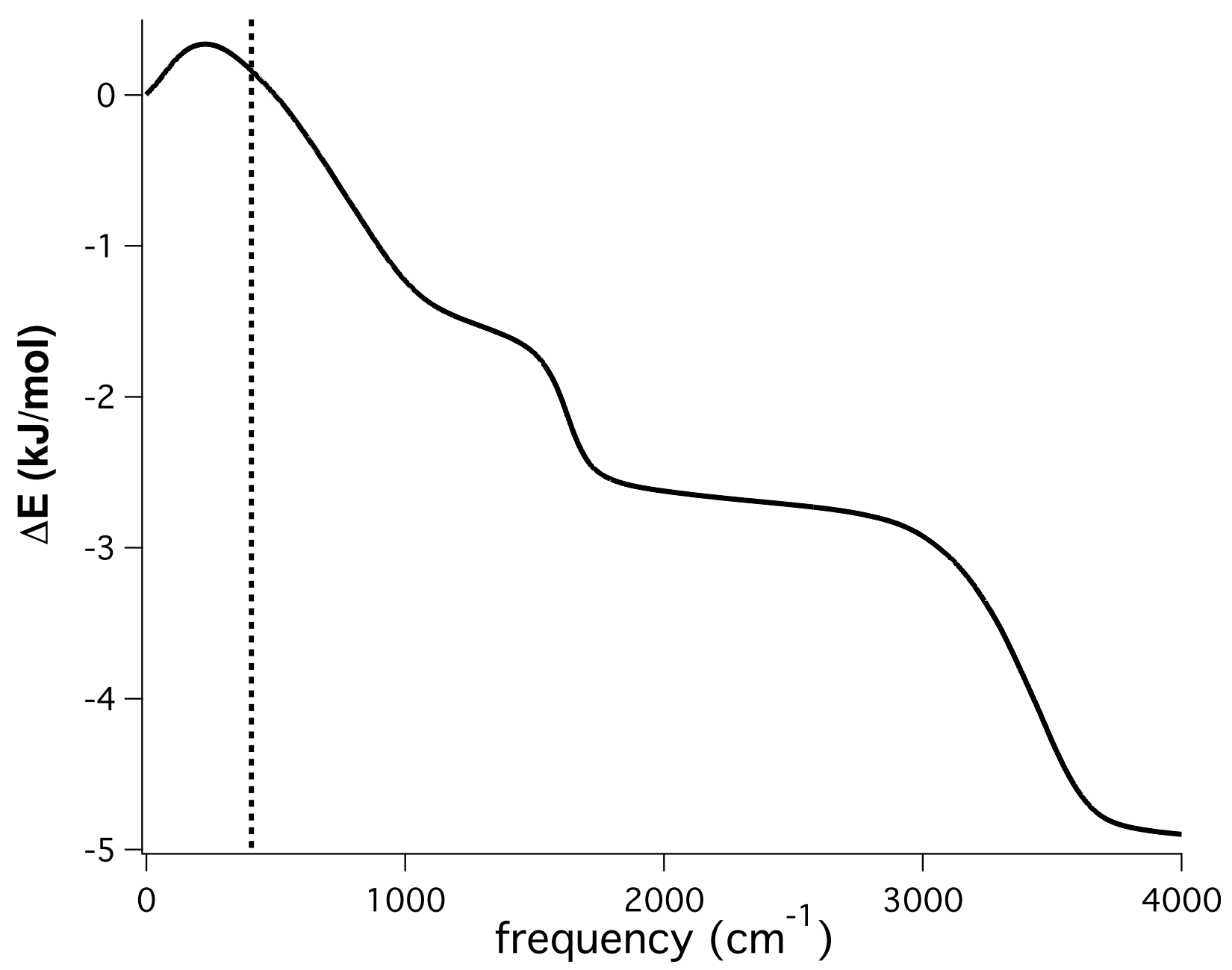

Figure 2: Plot of the difference between the quantum and classical thermal energies as a function of frequency for water at $T_{Q M}=584 \mathrm{~K}$ (dashed line). 


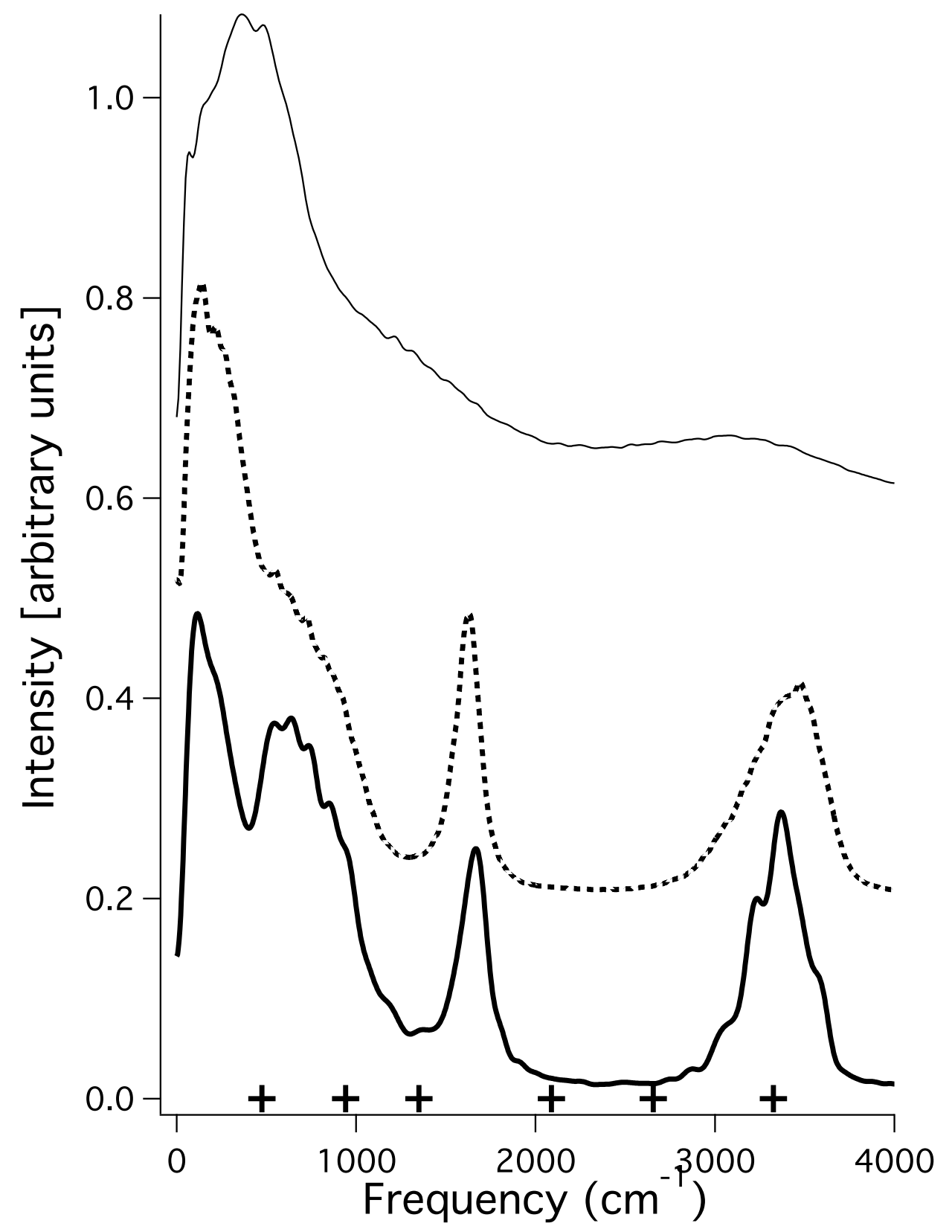

Figure 3: Plot of the power spectra of water at the initial conditions (300 K; thick black line), and quantum corrected temperatures $T_{Q M}$ of $584 \mathrm{~K}(5 \mathrm{~km} / \mathrm{s}$; dotted line), and $4244 \mathrm{~K}(11 \mathrm{~km} / \mathrm{s}$; thin black line). The ' + ' marks correspond to the $T_{Q M}$ values converted to wavenumbers. 


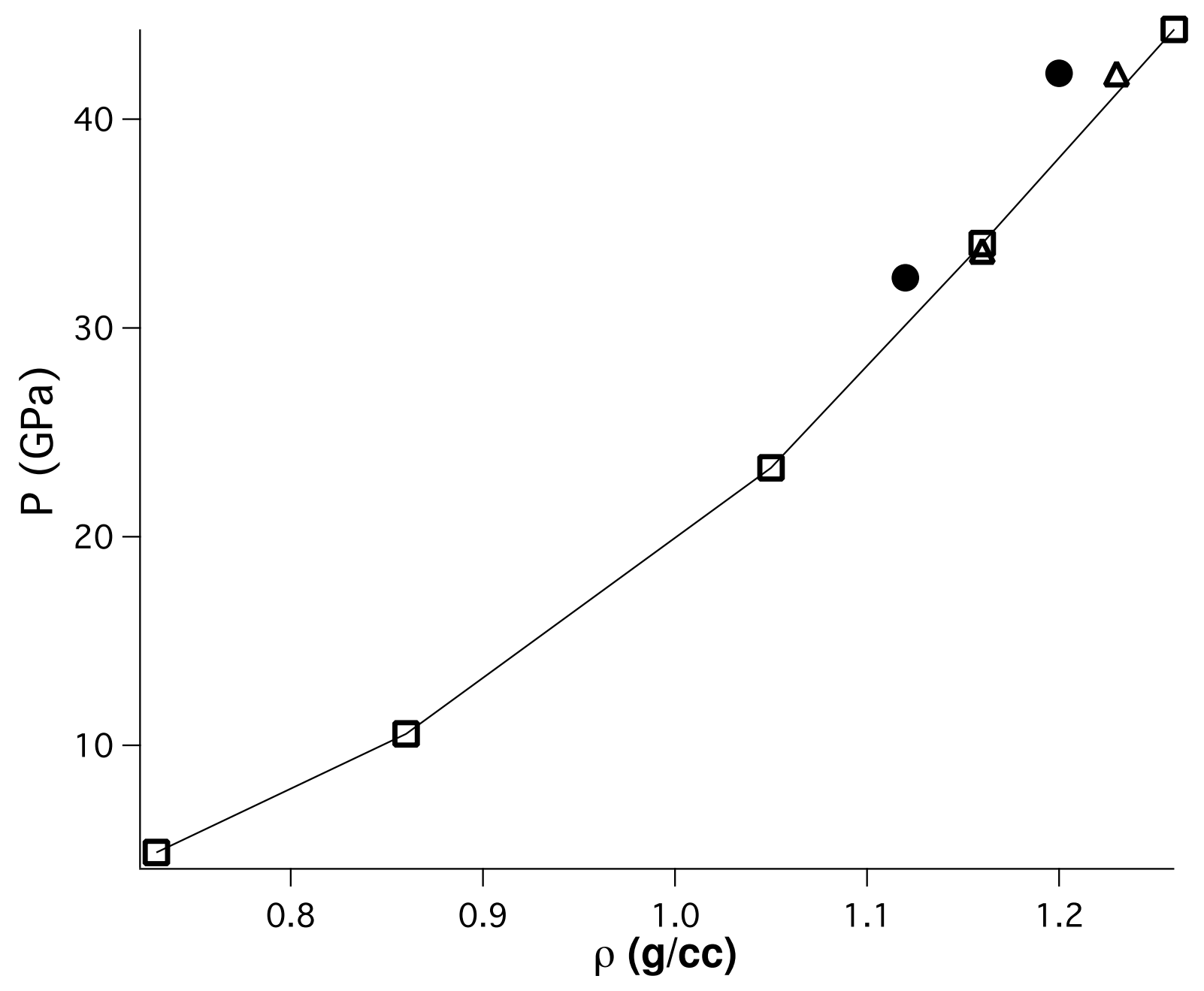

Figure 4: Plot of the pressure vs. density Hugoniot for $\mathrm{CH}_{4}$. The solid black circles correspond to our results form $\mathrm{CP} 2 \mathrm{~K}$, the open squares from Nellis et al. ${ }^{33}$ and the open triangles from Radousky et al. ${ }^{34}$ 


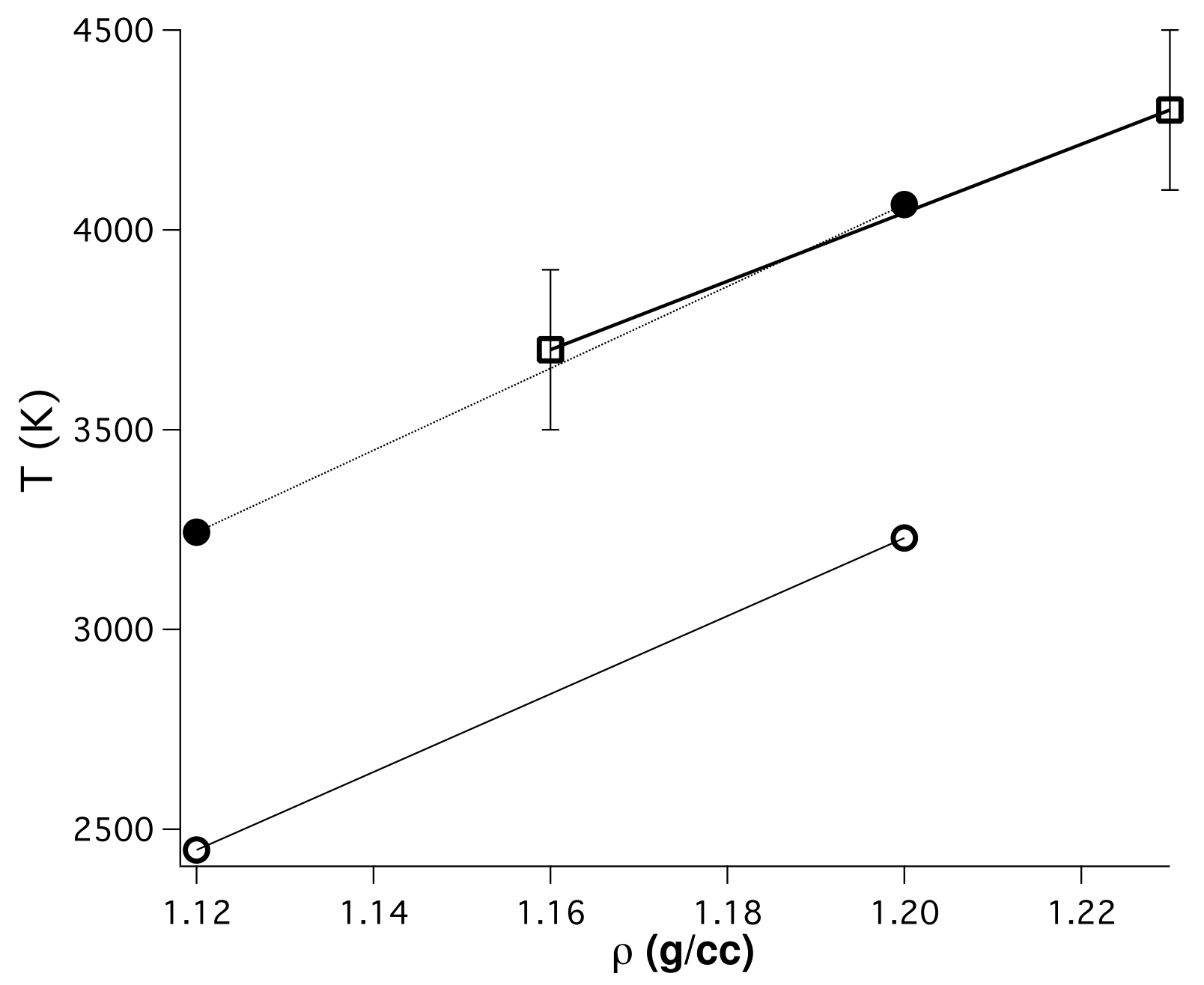

Figure 5: Plot of the temperature vs. density Hugoniot for $\mathrm{CH}_{4}$. The open circles correspond to the classical ionic temperature from $\mathrm{CP} 2 \mathrm{~K}$, the solid black circles to the quantum corrected temperatures, and the open sqaures with error bars to experimental results ${ }^{34}$. 


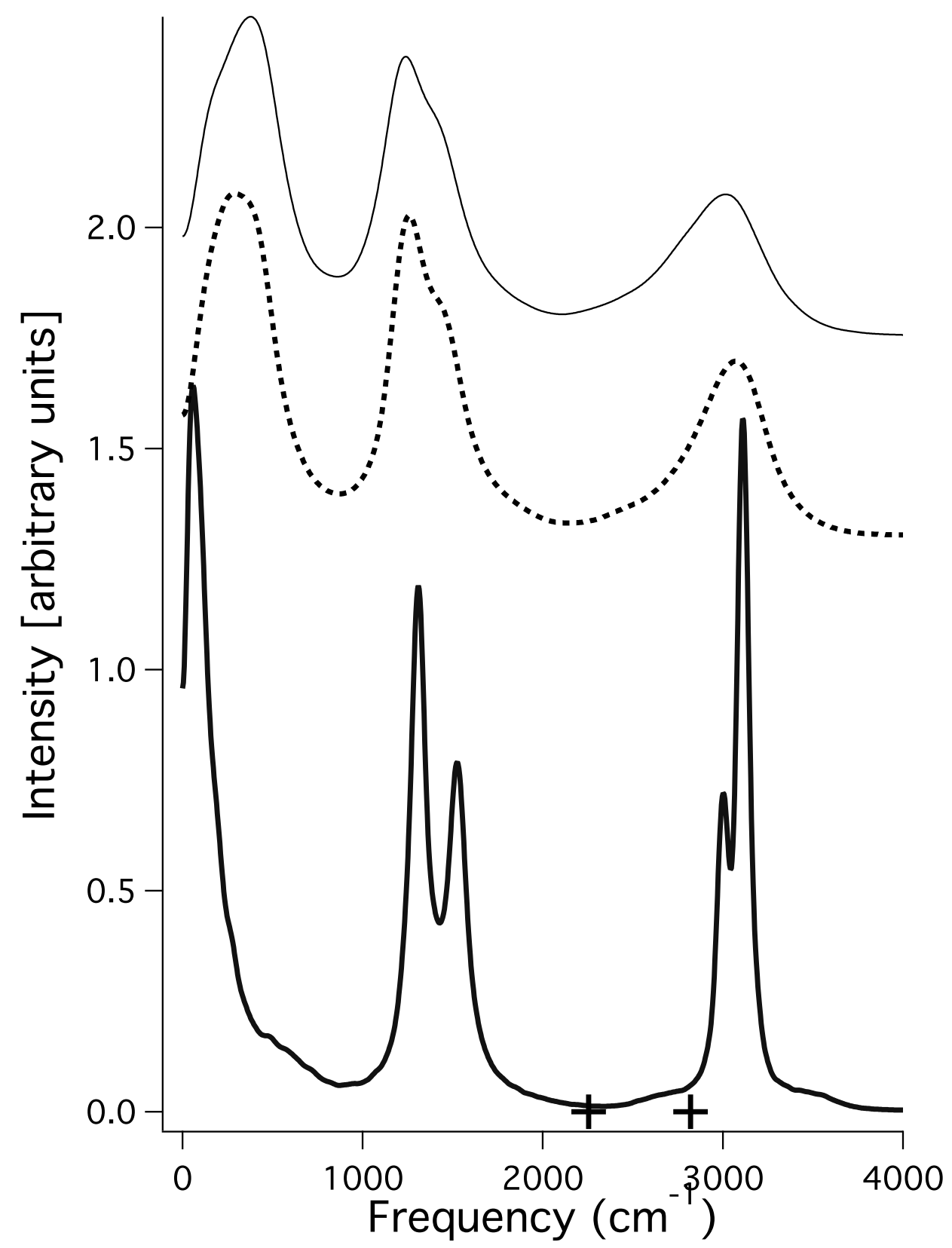

Figure 6: Plot of the vibrational density of states of methane at the initial conditions $(111 \mathrm{~K}$; thick black line), and $T_{Q M}$ values of $3244 \mathrm{~K}(11 \mathrm{~km} / \mathrm{s}$; dotted line), and $4059 \mathrm{~K}(12.2 \mathrm{~km} / \mathrm{s}$; thin black line). The '+' marks correspond to the $T_{Q M}$ values converted to wavenumbers. 